\title{
TALSPEAK Solvent Degradation
}

Leigh R. Martin

Bruce J. Mincher

September 2009

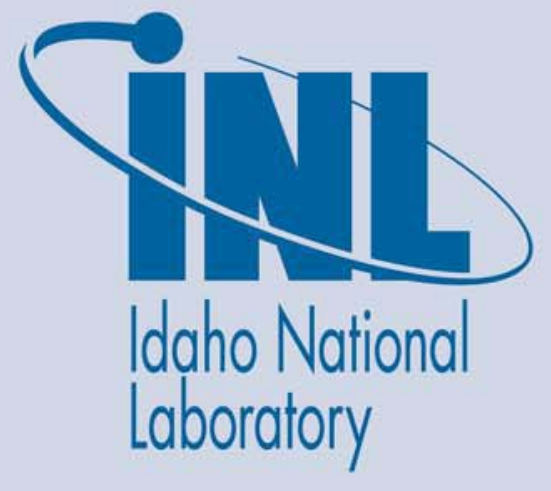

The INL is a U.S. Department of Energy National Laboratory operated by Battelle Energy Alliance 
INL/EXT-09-16893

\title{
TALSPEAK Solvent Degradation
}

\author{
Leigh R. Martin \\ Bruce J. Mincher
}

September 2009

\section{Idaho National Laboratory \\ Idaho Falls, Idaho 83415}

http://www.inl.gov

Prepared for the

U.S. Department of Energy Office of Nuclear Energy Under DOE Idaho Operations Office

Contract DE-AC07-05ID14517 


\section{TALSPEAK Solvent Degradation}

Advanced Fuel Cycle Initiative

Prepared for

U.S. Department of Energy Separations Campaign Leigh R. Martin and Bruce J. Mincher Idaho National Laboratory September $14^{\text {th }}, 2009$ AFCI-SEPA-PMO-MI-DV-2009-00188 


\section{DISCLAIMER}

This information was prepared as an account of work sponsored by an agency of the U.S. Government. Neither the U.S. Government nor any agency thereof, nor any of their employees, makes any warranty, expressed or implied, or assumes any legal liability or responsibility for the accuracy, completeness, or usefulness, of any information, apparatus, product, or process disclosed, or represents that its use would not infringe privately owned rights. References herein to any specific commercial product, process, or service by trade name, trade mark, manufacturer, or otherwise, does not necessarily constitute or imply its endorsement, recommendation, or favoring by the U.S. Government or any agency thereof. The views and opinions of authors expressed herein do not necessarily state or reflect those of the U.S. Government or any agency thereof. 


\section{SUMMARY}

Understanding the radiolytic degradation behavior of organic molecules involved in new or existing schemes for the recycle of used nuclear fuels is of significant interest for sustaining a closed fuel cycle. We have conducted several lines of investigation to begin understanding the effects of radiolysis on the aqueous phase of the TALSPEAK process for the separation of the trivalent lanthanides from the trivalent actinides. Using the ${ }^{60} \mathrm{Co}$ irradiator at the INL, we have begun to quantify the effects of radiation on the aqueous phase complexants used in this separation technique, and how this will affect the actinide lanthanide separation factor. In addition we have started to develop methodologies for stable product identification, a key element in determining the degradation pathways. We have also introduced a methodology to investigate the effects of alpha radiolysis that has previously received limited attention. 


\section{CONTENTS}

SUMMARY iii

ACRONYMS vi

1. INTRODUCTION.

2. SIGNIFICANCE.

3. APPROACH

3.1 Pulsed Radiolysis.

3.2 Static Irradiations.

Error! Bookmark not defined.

3.2.1 Solvent Extraction

Error! Bookmark not defined.

3.2.2 Stable Product Identification

Error! Bookmark not defined.

3.2.3

Alpha Radiolysis Error! Bookmark not defined.

4. SUMMARY OF RESULTS

Error! Bookmark not defined.

4.1 Pulsed Radiolysis

4.1.1 Nitrate radical reaction with lactic acid

Error! Bookmark not defined.

4.1.2 Hydroxyl radical reaction with DTPA. Error! Bookmark not defined.

4.2 Static Irradiations...

.3

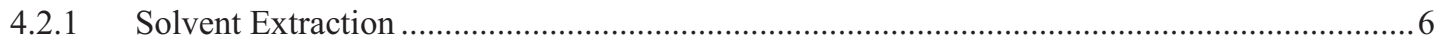

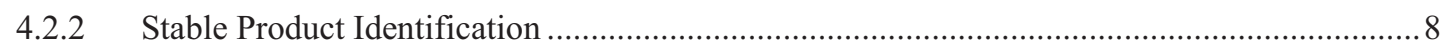

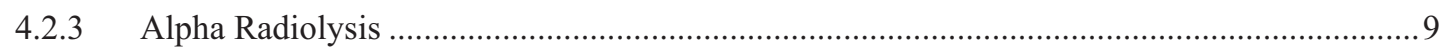

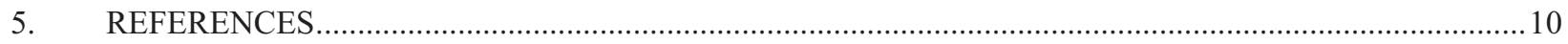

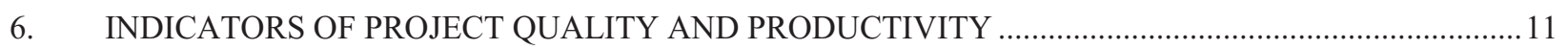

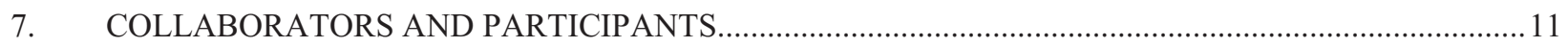

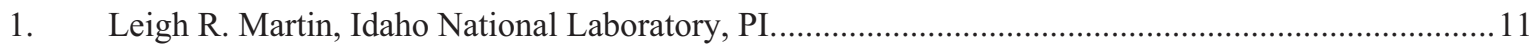

\section{FIGURES}

Figure 1. a) Decay of ${ }^{\circ} \mathrm{NO}_{3}$ radical at $640 \mathrm{~nm}$ in $\mathrm{N}_{2} \mathrm{O}$-saturated $6.0 \mathrm{M} \mathrm{HNO}_{3}$ with 0.141 (blue), 0.937 (green), 19.58 (red) and 65.57 (black) mM lactic acid added. b) Transformed second order plot of pseudo-first-order decay kinetics of a) plotted against lactic acid concentration. Solid line is weighted linear fit, corresponding to reaction rate constant of $k_{2}=2.15 \pm 0.17 \times 10^{5} \mathrm{M}^{-1} \mathrm{~s}^{-1}\left(\mathrm{R}^{2}=\right.$ 0.990).

Figure 2. Calculated speciation of DTPA based on given $\mathrm{pK}_{\mathrm{a}}$ values.

Figure 3. a) Transient absorbance of $(\mathrm{SCN})_{2}{ }^{-}$from $\mathrm{N}_{2} \mathrm{O}$-saturated $118.6 \mu \mathrm{M} \mathrm{KSCN}$ solution containing zero (black), 61.4 (red), 119.3 (green), 182.8 (blue) and 252.7 (magenta) $\mu$ M DTPA at pH 3.12 should you really give all these digits for a $\mathrm{pH}$ measurement?. b) Transformed competition kinetics plot from data of a). Solid red line corresponds to weighted linear fit, with intercept of $0.99072 \pm 0.009$ and slope of $0.2694 \pm 0.007$ same comment, corresponding to a rate constant for ${ }^{\bullet} \mathrm{OH}$ reaction with DTPA of $3.13 \pm 0.08 \times 10^{9} \mathrm{M}^{-1} \mathrm{~s}^{-1}$ at this $\mathrm{pH}\left(\mathrm{R}^{2}=0.998\right)$.

Figure 4 Distribution ratios for Eu and Am versus absorbed dose to lactic acid only for TALSPEAK extractions using $0.17 \mathrm{M}$ pure and non-purified HDEHP. Error bars shown are standard deviations of triplicate measurements. 
Figure 5 Distribution ratios for Eu and Am versus absorbed dose to DTPA only for TALSPEAK extractions using $0.17 \mathrm{M}$ pure and non-purified HDEHP. Error bars shown are standard deviations of triplicate measurements.

Figure 6 Distribution ratios for Eu and Am versus absorbed dose to Lactic acid/ DTPA only for TALSPEAK extractions using 0.17M pure and non-purified HDEHP. Error bars shown are standard deviations of triplicate measurements.

Figure 7 HPLC diode array chromatograms for lactate buffer $\mathrm{pH} 3.8,(-)$ with no irradiation and (一) after being irradiated to $200 \mathrm{kGy}$.

Figure 8. Sample cell after irradiation of $1 \times 10^{-3} \mathrm{M}$ sodium lactate at $\mathrm{pH} 3.7$ for 30 mins. The generated gas is unidentified.

\section{TABLES}

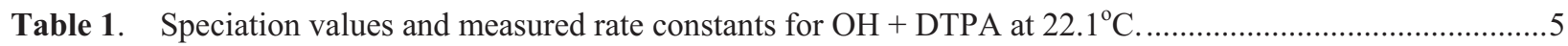

Table 2. Calculated individual rate constants for acidic DTPA species. 


\section{ACRONYMS}

CNRS

D

DTPA

FID

HDEHP

HPLC

HPLC-MS

INL

LINAC

NMR

PNNL

TALSPEAK

UNLV

US DOE

UV-vis
Centre National de la Rescherche Scientifique

Distribution coefficient

Di 2-ethylenetriamine-N,N,N',N",N"-pentaacetic acid

Flame Ionization Detection

Di ethyl hexyl phosphoric acid

High Performance Liquid Chromatography

High Performance Liquid Chromatography - Mass Spectrometry

Idaho National Laboratory

Linear Accelerator

Nuclear Magnetic Resonance

Pacific Northwest National Laboratory

Trivalent Actinide Lanthanide Separations by Phosphorus-reagent Extraction from Aqueous Komplexes

University of Nevada Las Vegas

United States Department of Energy

Ultra Violet visible 
TALSPEAK Solvent Degradation

September $14^{\text {th }}, 2009$ 



\section{SEPARATIONS CAMPAIGN}

\section{TALSPEAK SOLVENT DEGRADATION}

\section{INTRODUCTION}

Although the TALSPEAK process ${ }^{1,2}$ is at an advanced development stage, there is still little information available on the radiation resistance of the complexants used to accomplish the separation. ${ }^{3}$ Predictability of system performance under "off normal" conditions is also an important criterion for the development of production-scale separation processes. This is best achieved through a thorough understanding of the chemistry of not only the various metal-ligand-solvent interactions that contribute to the net separation but also the degradation products from the radiolysis or hydrolysis of the extraction process components. The production of even small quantities of radiolytic degradation products of these complexants may result in significant effects on the distribution of the both $\mathrm{An}^{3+}$ and $\mathrm{Ln}^{3+}$ ions.

We have investigated the effects of radiolysis on the aqueous phase components of the TALSPEAK process to try to determine what stable products are produced and, hence, determine mechanisms for the degradation pathways from gamma radiolysis. In addition to this study, we completed a short investigation using an alpha ion beam to identify if similar products would be observed. In tandem with the static irradiations for stable product identification, we have conducted batch solvent extraction experiments to identify the effects of gamma radiolysis on separation factors of americium and europium when irradiating lactate and DTPA separately and then lactate and DTPA in solution together. Initial pulse radiolysis experiments have also been performed on other components of the system.

\section{SIGNIFICANCE}

Understanding the radiolytic degradation behavior of organic molecules involved in new or existing schemes for the recycling of used nuclear fuels is of significant interest for sustaining a closed nuclear fuel cycle. The high radiation fields that exist in dissolved nuclear fuels, and raffinates from processed fuel, produce a variety of transient and long-lived species which react with the reagents that facilitate these separations. Little information is available on the radiation resistance of the aqueous phase complexants of the TALSPEAK process; lactic acid and diethylenetriamine-N,N,N',N",N"-pentaacetic acid (DTPA), used to accomplish the separation.

\section{APPROACH}

Previously funded LDRD work at INL identified that the lactic acid buffer in TALSPEAK somewhat reduces the oxidative damage to DTPA from hydroxyl radical reactions. However, its relatively slow reaction rate constant does not prevent the DTPA from being degraded during the process. ${ }^{4}$ Developing on these ideas, we have used several techniques to attempt to further understand degradation mechanisms of lactic acid and the resulting effects on the TALSPEAK process. These methodologies are discussed below.

\subsection{Pulsed Radiolysis, e-beam irradiations}

To determine if there were any other reactive species in aqueous solution that might degrade the lactic acid we conducted pulsed radiolysis experiments at the US DOE Notre Dame Radiation Laboratory. The equipment and facility have been described elsewhere. ${ }^{4,5}$ As the $\mathrm{pH}$ of the TALSPEAK aqueous phase will be adjusted to $\sim 3.8$, significant quantities of nitrate will most likely be present; therefore, the investigations focused on determining the rate of reaction of the nitrate radical with lactic acid. In addition to these studies, we began to look at the reaction of the hydroxyl radical with DTPA. 


\section{2 ${ }^{60}$ Co Static Gamma Irradiations}

\subsubsection{Solvent Extraction \& Stable Product Identification}

Static irradiations on TALSPEAK process aqueous phases were performed to investigate the effects of irradiating the individual components (lactate buffer, DTPA and a lactate buffer:DTPA mix) under TALSPEAK relevant conditions. These aqueous phases were then spiked with tracer americium and europium and solvent extractions were performed to quantify how the effects of increasing radiation dose would change the performance of the solvent extraction system. The effects of purified HDEHP vs non purified HDEHP solvent were also studied. Finally, we investigated stripping of the metals from the loaded solvent.

For completeness in the determination of the radiolysis degradation mechanisms, stable product identifications are very important. To that end, the products of lactate buffer irradiation (high and low concentration) were investigated by high performance liquid chromatography (HPLC) with flame ionization detection (FID). The chromatograms were compared to known samples in an attempt to identify the final stable products. This work was performed at both INL and University of Nevada Las Vegas (UNLV).

\subsection{Alpha Radiolysis}

There has been no previous work performed on the alpha radiolysis of lactic acid and DTPA. We have conducted initial investigations using a $5 \mathrm{MeV}$ He ion beam to probe the effects of alpha radiolysis on lactic acid/ lactate and HDEHP. This investigation was primarily to look for degradation products formed.

\section{SUMMARY OF RESULTS}

\subsection{Pulsed Radiolysis}

\subsubsection{Nitrate radical reaction with lactic acid}

To generate the nitrate radicals required for this study, fast pulses of accelerator generated electrons were delivered to a series of $6 \mathrm{M}$ nitric acid solutions containing various concentrations of lactic acid. The nitrate radicals themselves result from one of two mechanisms, either the direct radiolysis of $\mathrm{HNO}_{3}$ or by the reaction with hydroxyl radical, Equations $1 \& 2:^{5}$

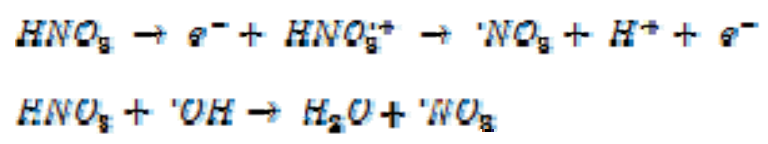

$$
k_{1}=1.4 \times 10^{8} \mathrm{M}^{-1} \mathrm{~s}^{-1}
$$

The decay of the UV-vis absorbance of the nitrate radical was observed over a microsecond timescale (Figure 1a) to determine pseudo first order decay rates. These first order decay rates were then plotted vs lactic acid concentration (Figure 1b). The slope of the linear fit corresponds to the second order rate constant $\left(k_{2}\right)$ which was determined to be $2.15 \pm 0.17 \times 10^{5} \mathrm{M}^{-1} \mathrm{~s}^{-1}$. The slow rate constant for this reaction indicates that ${ }^{\bullet} \mathrm{NO}_{3}$ radical reaction with lactic acid will not be a significant contributor to the degradation of lactic acid in the TALSPEAK system. This being the case, it is not expected that there will be any reactions with lactic acid and any ${ }^{\bullet} \mathrm{NO}_{2}$ radical that could be generated in this system as generally these rates are even slower than ${ }^{\circ} \mathrm{NO}_{3}$ radical reactions. When we also consider that these separations systems will contain significant amounts of dissolved oxygen and are mildly acidic we can rule out any significant contribution to degradation from the hydrated electron and hydrogen atoms as they are both rapidly consumed, Equations $3 \& 4:^{6-8}$ 


$$
\begin{aligned}
& \theta_{(a q)}+H^{+}-H^{\prime} \\
& H^{\prime}+O_{2}-H O_{2}^{\prime}
\end{aligned}
$$

$$
\begin{aligned}
k_{3}= & 2.3 \times 10^{10} \mathrm{M}^{-1} \mathrm{~s}^{-1} \\
& k_{4}=1.2 \times 10^{10} \mathrm{M}^{-1} \mathrm{~s}^{-1}
\end{aligned}
$$

This leaves the ${ }^{\bullet} \mathrm{OH}$ radical as the only transient radiolysis product that could be responsible for the degradation of lactic acid and lactate ions in TALSPEAK.
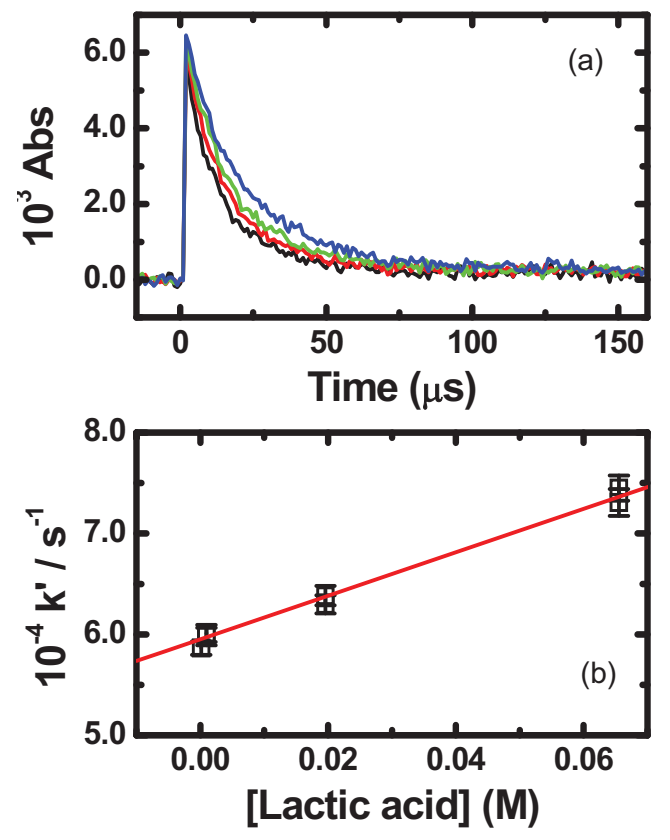

Figure 1. a) Decay of ${ }^{\circ} \mathrm{NO}_{3}$ radical at $640 \mathrm{~nm}$ in $\mathrm{N}_{2} \mathrm{O}$-saturated $6.0 \mathrm{M} \mathrm{HNO}_{3}$ with 0.141 (blue), 0.937 (green), 19.58 (red) and 65.57 (black) mM lactic acid added. b) Transformed second order plot of pseudo-first-order decay kinetics of a) plotted against lactic acid concentration. Solid line is weighted linear fit, corresponding to reaction rate constant of $k_{2}=2.15 \pm 0.17 \times 10^{5} \mathrm{M}^{-1} \mathrm{~s}^{-1}\left(\mathrm{R}^{2}=0.990\right)$.

\subsubsection{Hydroxyl radical reaction with DTPA}

The other major constituent of TALSPEAK is DTPA. Currently there are no existing studies of hydroxyl radical reactions with DTPA under acidic conditions, although reaction rates have been measured at neutral $\mathrm{pH}$ 's $(\mathrm{k} \approx 5.2 \mathrm{x}$ $\left.10^{9} \mathrm{M}^{-1} \mathrm{~s}^{-1}\right) \cdot{ }^{9,10}$ One of the difficulties in making these measurements with DTPA in comparison to lactic acid and the lactate ion is that over the $\mathrm{pH}$ range 1-12 there are 6 separate species to be considered (Figure 2). In the operating envelope of TALSPEAK at a $\mathrm{pH} 2-4$, there are 4 species that probably need to be considered, each with their own individual reaction rate constant. The species to be considered are $\mathrm{H}_{5}$ DTPA, $\mathrm{H}_{4} \mathrm{DTPA}, \mathrm{H}_{3}$ DTPA and $\mathrm{H}_{2} \mathrm{DTPA}$, with $\mathrm{H}_{3}$ DTPA being the most important. 


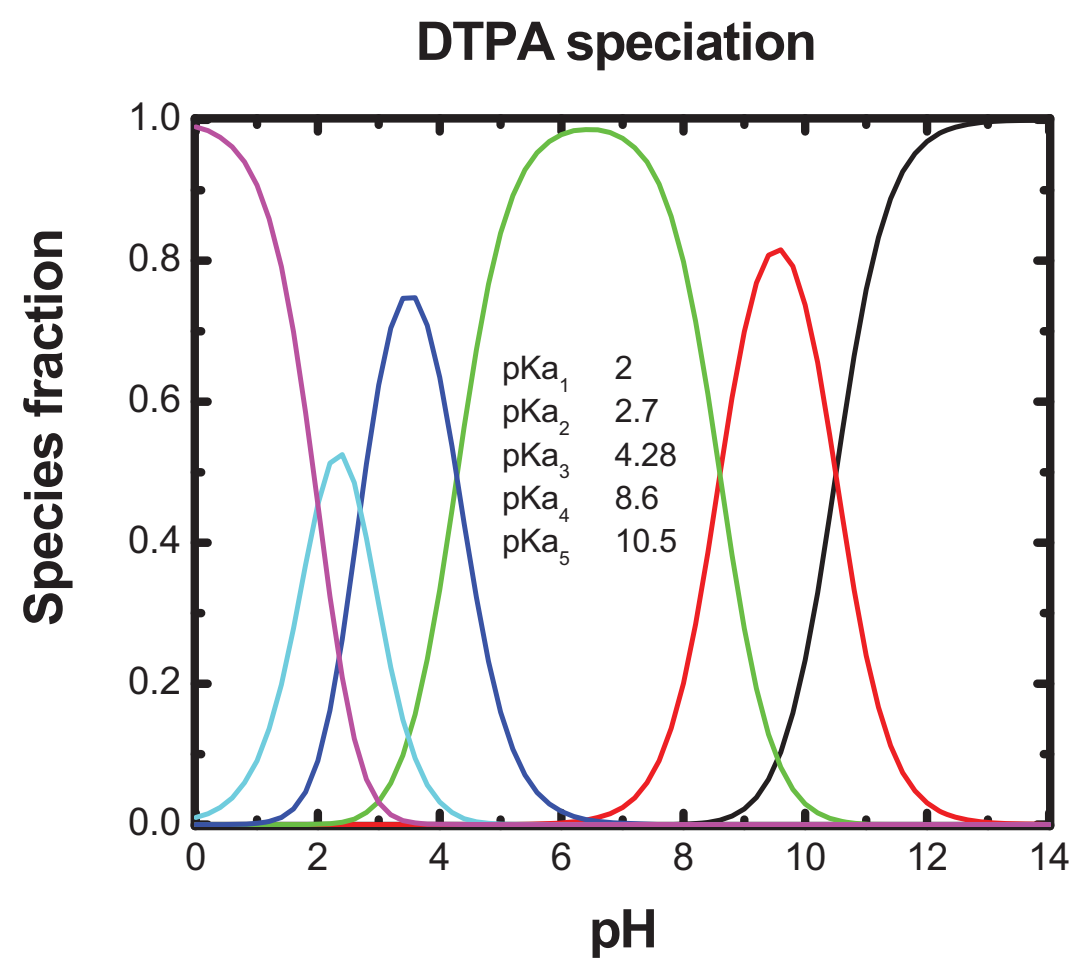

Figure 2. Calculated speciation of DTPA based on given $\mathrm{pK}_{\mathrm{a}}$ values.

An experiment to determine the ${ }^{\circ} \mathrm{OH}$ radical reaction rate with the DTPA species in solution was carried out in a similar manner to described in section 4.1 .1 for the ${ }^{\circ} \mathrm{NO}_{3}$ radical. ${ }^{45}$ Briefly, the ${ }^{\bullet} \mathrm{OH}$ radical reaction rate constants were determined using the linear accelerator (LINAC) electron pulse radiolysis system at the US DOE Notre Dame Radiation Laboratory. Solutions of DTPA were prepared at pH's of 1.22, 2.00 and 3.12 and sparged with either high purity $\mathrm{O}_{2}$ or high purity $\mathrm{N}_{2} \mathrm{O}$ to remove any hydrated electrons or hydrogen atoms generated from solution. There is no direct useful transient absorbance for the ${ }^{\bullet} \mathrm{OH}$ radical in aqueous solution; therefore, the rate constants were determined by $\mathrm{SCN}^{-}$competition kinetics, monitoring the decrease in $(\mathrm{SCN})_{2}{ }^{\bullet-}$ transient at $475 \mathrm{~nm}$ with increasing DTPA concentration (Figure 3a). By plotting $1 / \mathrm{Abs}(\mathrm{SCN})_{2}{ }^{\bullet-}$ vs $[\mathrm{DTPA}] /\left[\mathrm{SCN}^{-}\right]$a straight line is obtained where the slope corresponds to Equation 5:

\section{slope $=\frac{\text { rate of ' } O H \text { reacton with DTFA }}{\text { rate of 'OH reacton with SCN }}$}

since the rate of ${ }^{\bullet} \mathrm{OH}$ radical reaction with SCN- is known $\left(k=1.05 \times 10^{10} \mathrm{M}^{-1} \mathrm{~s}^{-1}\right)^{6}$ then the value of the ${ }^{\bullet} \mathrm{OH}$ radical reaction with DTPA can be determined. 

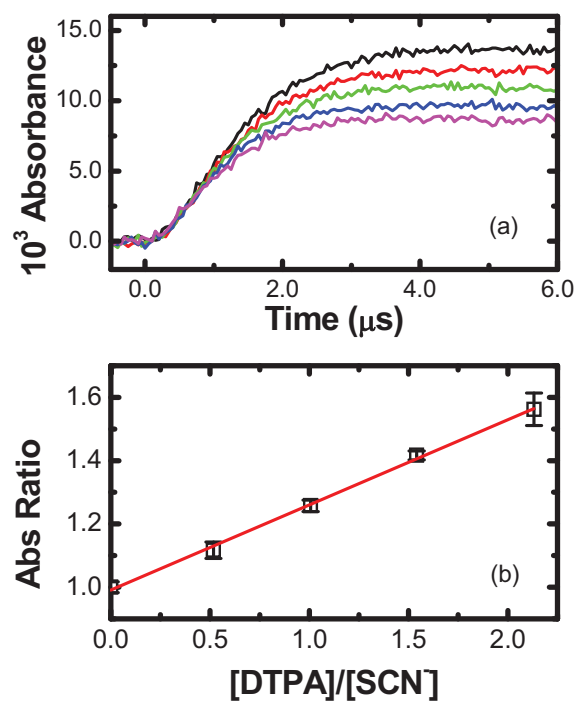

Figure 3. a) Transient absorbance of $(\mathrm{SCN})_{2}{ }^{-}$from $\mathrm{N}_{2} \mathrm{O}$-saturated $118.6 \mu \mathrm{M} \mathrm{KSCN}$ solution containing zero (black), 61.4 (red), 119.3 (green), 182.8 (blue) and 252.7 (magenta) $\mu$ M DTPA at pH 3.12. b) Transformed competition kinetics plot from data of a). Solid red line corresponds to weighted linear fit, with intercept of 0.99072 \pm 0.009 and slope of $0.2694 \pm 0.007$ same comment, corresponding to a rate constant for ${ }^{\circ} \mathrm{OH}$ reaction with DTPA of $3.13 \pm 0.08 \times 10^{9} \mathrm{M}^{-1} \mathrm{~s}^{-1}$ at this $\mathrm{pH}\left(\mathrm{R}^{2}=0.998\right)$.

The initial experimental reaction rate measurements and individual rate constants for $\mathrm{H}_{5} \mathrm{DTPA}, \mathrm{H}_{4} \mathrm{DTPA}$, $\mathrm{H}_{3}$ DTPA are listed in Tables 1 and 2.

Table 1. Speciation values and measured rate constants for $\mathrm{OH}+\mathrm{DTPA}$ at $22.1^{\circ} \mathrm{C}$.

\begin{tabular}{c|c|c|c|c}
\hline $\mathbf{p H}$ & $\begin{array}{c}\mathbf{H}_{3} \text { DTPA } \\
\text { (blue) }\end{array}$ & $\begin{array}{c}\mathbf{H}_{4} \text { DTPA } \\
\text { (cyan) }\end{array}$ & $\begin{array}{c}\mathbf{H}_{5} \text { DTPA } \\
\text { (pink) }\end{array}$ & $\begin{array}{c}\text { OH + DTPA k values } \\
\mathbf{M}^{-1} \mathbf{s}^{-1}\end{array}$ \\
\hline 3.12 & 0.6765 & 0.2572 & 0.01951 & $3.13 \pm 0.08 \times 10^{9}$ \\
2.000 & 0.09067 & 0.4544 & 0.4544 & $3.06 \pm 0.06 \times 10^{9}$ \\
1.218 & 0.004651 & 0.1411 & 0.8542 & $2.79 \pm 0.11 \times 10^{9}$ \\
\hline
\end{tabular}

Table 2. Calculated individual rate constants for acidic DTPA species.

\begin{tabular}{c|c}
\hline Species & OH + DTPA k values \\
& $M^{-1} \mathrm{~s}^{-1}$ \\
\hline H$_{3}$ DTPA (blue) & $3.26 \times 10^{9}$ \\
H$_{4}$ DTPA (cyan) & $3.40 \times 10^{9}$ \\
H$_{5}$ DTPA (pink) & $2.69 \times 10^{9}$ \\
\hline
\end{tabular}

The rate constants determined were found to be slightly slower than the $\mathrm{k} \approx 5.2 \times 10^{9} \mathrm{M}^{-1} \mathrm{~s}^{-1}, 9,10$ measured at higher $\mathrm{pH}$. As there is no clear trend in the rate of reaction with regards to the protonation of the DTPA ligand itself it is currently difficult to speculate on reaction mechanisms with the ${ }^{\bullet} \mathrm{OH}$ radical, additional information from steady state experiments will be required before any mechanistic information could be considered.

Future investigations will continue to determine accurate rate constants for the DTPA species relevant to the TALSPEAK process and also to determine the temperature dependent rate constants for these reactions. In addition the effects of metal complexation on ${ }^{\bullet} \mathrm{OH}$ radical reaction rates will be examined. 


\section{$4.2{ }^{60}$ Co Static Gamma Irradiations}

\subsubsection{Solvent Extraction}

Lactic acid buffered to $\mathrm{pH} 3.8$ was initially irradiated in the absence of any DTPA and organic phase to doses of $5,10,100$ and $200 \mathrm{kGy}$. After the irradiation DTPA was added to a concentration of $50 \mathrm{mM}$ (as expected in TALSPEAK) and then these solutions were used to perform solvent extraction experiments with tracer americium and europium. The organic phase for these extractions was $0.17 \mathrm{M}$ Diethyl hexyl phosphoric acid (HDEHP). Two sets of experiments were performed, one with an organic phase containing purified HDEHP and one with an organic phase containing non-purified HDEHP. The resulting plots of distribution ratio vs. absorbed dose are shown in Figure 1.
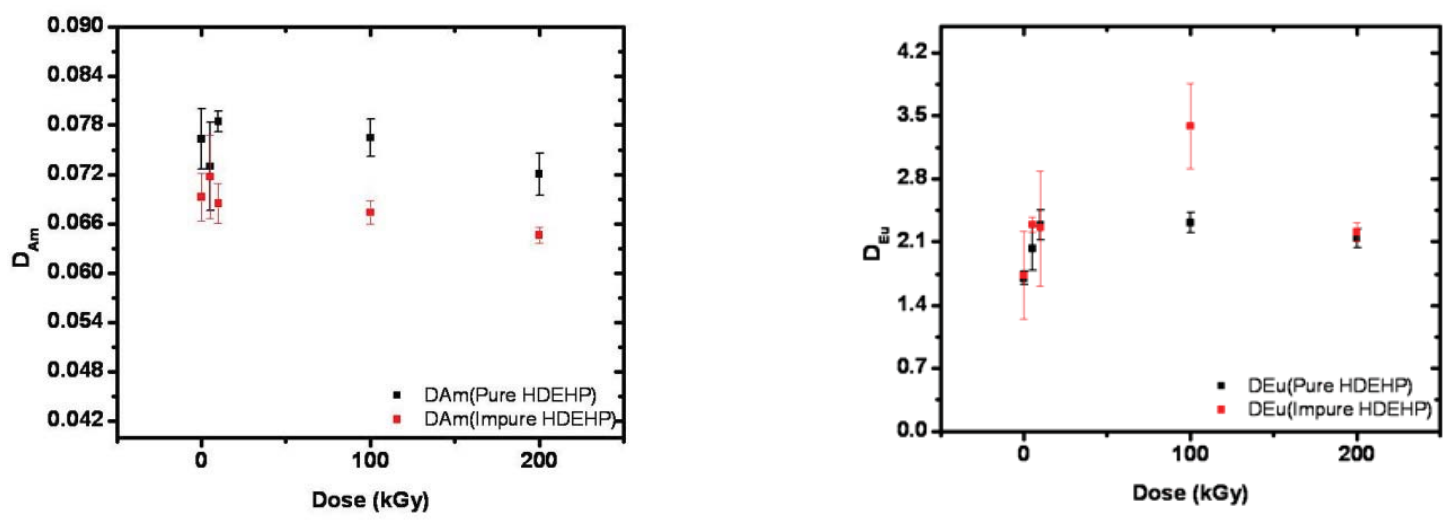

Figure 4 Distribution ratios for Eu and Am versus absorbed dose to lactic acid only for TALSPEAK extractions using $0.17 \mathrm{M}$ pure and non-purified HDEHP. Error bars shown are standard deviations of triplicate measurements.

The data shown in Figure 4 shows that essentially no americium is extracted however, the D values for what americium is extracted does show a small decrease with increasing absorbed dose. For europium the distribution coefficient is seen to increase with increasing dose to lactic acid. These results are somewhat interesting as the different trends in the distribution coefficient with increasing dose would seem to suggest that lactate plays a different role in the extraction of americium and europium.

When comparing the results of pure vs non-purified HDEHP as the extractant we found that there was very little difference in the observed trend in extraction profile. Therefore, we proceeded to use non purified HDEHP in dodecane for the rest of the solvent extraction experiments that were conducted.

Solutions of DTPA at a concentration of $0.1 \mathrm{M}$ and a pH 3.8 were initially irradiated in the absence of any lactic acid and organic phase to doses of 5, 10, 100 and $200 \mathrm{kGy}$. Post irradiation, non-irradiated lactic acid was added to the DTPA solutions to a concentration of $1.0 \mathrm{M}$ (as expected in TALSPEAK). Similarly to the initial experiment, these solutions were used to perform solvent extraction experiments with tracer americium and europium. As reported above, with little difference in the behavior of the pure vs non-purified HDEHP solvent only non-purified $0.17 \mathrm{M}$ HDEHP organic phase was used in these investigations. The resulting plots of distribution ratio vs. absorbed dose are shown in Figure 5.

It is clear comparing Figures 4 and 5 that irradiating DTPA only has a significantly different effect on the results of the solvent extraction experiments for americium and europium. For both $\mathrm{Am}$ and $\mathrm{Eu}$, the trend illustrated by Figure 5 shows an increase in solvent extraction efficiency for both elements, contrary to the lactate results. This is not to be unexpected as the role of DTPA in TALSPEAK is to retain the actinides in the aqueous phase, therefore if we degrade DTPA one would expect americium and europium extractions to increase. Total europium extracted 
is seen to increase from $60 \%$ to $80 \%$ while total americium extracted increases from 2 to $5 \%$. Even though these increases seem significantly different the separation factor of $\mathrm{Eu}$ from Am only increases from 71 to 80 . What is more important is that when stripping tests were conducted on these organic phases with $2 \mathrm{M}$ nitric acid, no metal was retained in the organic phase. These results suggest that it is unlikely that the degradation products play any role in the extracted complexes, or if they do they do not interfere with the stripping chemistry. An additional point of note is that the majority of DTPA degradation products are almost certainly amino polycarboxylic acids. This being the case, these species can also interact with Am and Eu in the same manner as DTPA under TALSPEAK conditions and hence we don't see a more dramatic change in the separation performance at higher doses.
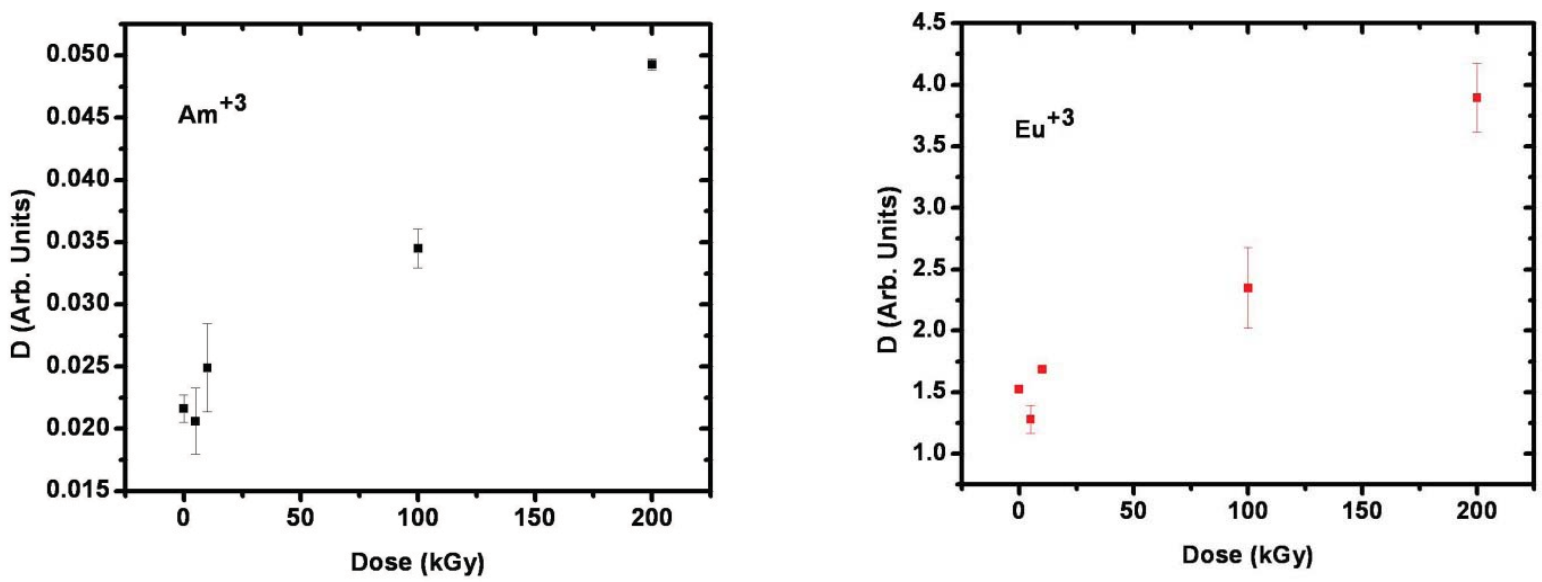

Figure 5 Distribution ratios for Eu and Am versus absorbed dose to DTPA only for TALSPEAK extractions using $0.17 \mathrm{M}$ pure and non-purified HDEHP. Error bars shown are standard deviations of triplicate measurements.

The final set for solutions that were irradiated for solvent extraction batch contact experiments consisted of 0.05 M DTPA in $1.0 \mathrm{M}$ lactic acid at $\mathrm{pH} 3.8$, as expected in the TALSPEAK formulation. Once again these solutions were irradiated in the ${ }^{60} \mathrm{Co}$ source at the INL to final doses of 5, 10, 100 and $200 \mathrm{kGy}$ in the absence of any organic phase. Post irradiation, these solutions were spiked with radiotracer americium and europium and contacted with non-purified 0.17 M HDEHP. The resulting plots of distribution ratio vs. absorbed dose are shown in Figure 6.

The results from these tests show that irradiating a mix of lactic acid and DTPA has a similar effect on the distribution coefficients as irradiating DTPA only. However, the observed distribution coefficients do not increase to the same degree. This is due to the fact that in TALSPEAK the major species responsible for the degradation of both lactic acid and DTPA is most likely the ${ }^{\bullet} \mathrm{OH}$ radical.

Using our experimentally determined rate constants for the reaction of ${ }^{\bullet} \mathrm{OH}$ radical with lactic acid, the lactate anion and $\mathrm{H}_{3} \mathrm{DTPA}$, a relative rates analysis shows that approximately $80 \%$ of the ${ }^{\circ} \mathrm{OH}$ radical reacts with the lactate buffer, meaning that the remaining $20 \%$ reacts with the DTPA. Thus, the presence of lactic acid protects DTPA from ${ }^{\circ} \mathrm{OH}$ radical reactions, resulting in a less dramatic effect on the $\mathrm{D}_{\mathrm{Am}}$ and $\mathrm{D}_{\mathrm{Eu}}$. Despite this encouraging result, as DTPA is the ligand that is essentially responsible for the separation we would like to see more protection of this species from radiolysis.

We will continue to investigate the effects of gamma radiolyis on the individual and combined components of the TALSPEAK system. To further our understanding we need to investigate the gamma radiolysis effects on the organic phase of HDEHP in dodecane, and the resultant effects on the extraction of americium and europium. In addition we will also need to perform investigations into the behavior of $\mathrm{Cm}$ in this system. Ultimately we will also need to investigate the effects of gamma radiolysis when mixing the organic and aqueous phases, after the individual static systems are understood. 

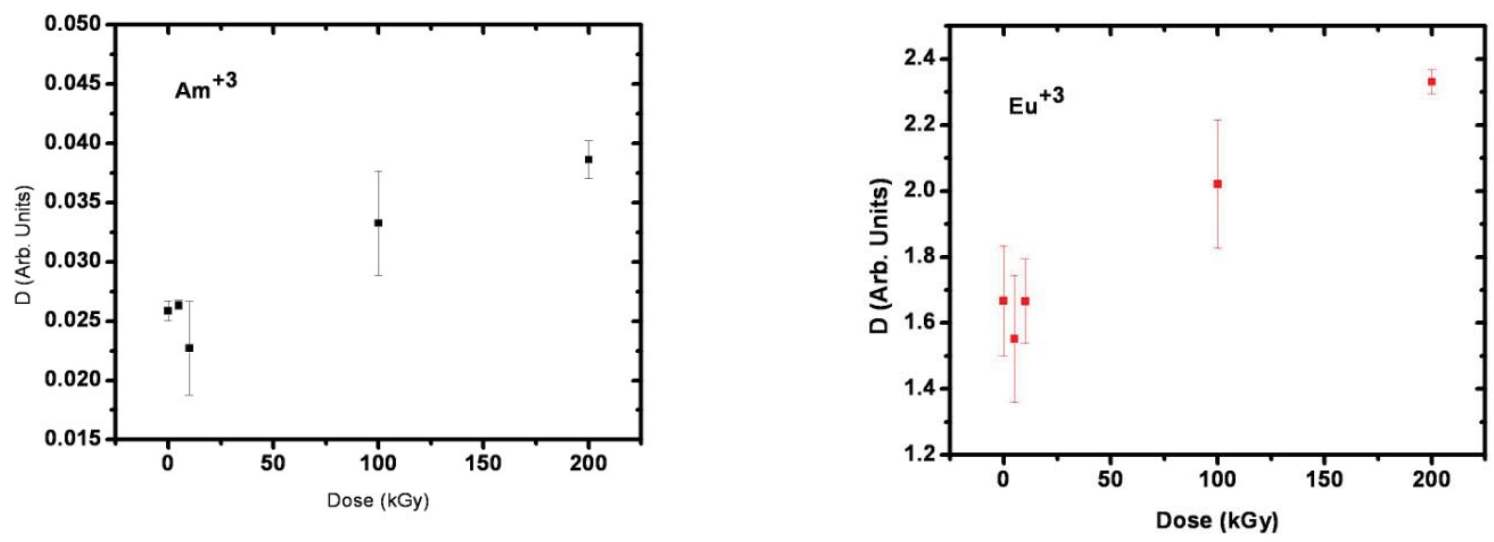

Figure 6 Distribution ratios for Eu and Am versus absorbed dose to Lactic acid/ DTPA only for TALSPEAK extractions using $0.17 \mathrm{M}$ pure and non-purified HDEHP. Error bars shown are standard deviations of triplicate measurements.

\subsubsection{Stable Product Identification}

To assist in understanding the degradation mechanism of lactic acid and DTPA we have begun method development for determining stable products after irradiation of lactic acid and lactate buffer to 5, 10, 100 and 200 kGy. To do this, a Shimadzu HPLC with photodiode array detector was used for the analysis of lactate. Biorad's Aminex HPX-87-H2 ion exclusion column $(300 \times 7.8 \mathrm{~mm})$ was used for the separation with $0.01 \mathrm{~N}$ sulfuric acid as the solvent. The column temperature was maintained at $50^{\circ} \mathrm{C}$ and the column was equilibrated for one hour at 0.2 $\mathrm{ml} / \mathrm{min}$. flow rate. The flow rate was then gradually increased to $0.3,0.4,0.5$, up to a final flow rate of $0.6 \mathrm{ml} / \mathrm{min}$. An injection volume of $10 \mu \mathrm{l}$ was used and the chromatograms were obtained at $210 \mathrm{~nm}$. The retention time of lactate in this condition was $\sim 13 \mathrm{~min}$.

Irradiated and non-irradiated lactate samples of concentration $0.001 \mathrm{M}$ and $1 \mathrm{M}$ at $\mathrm{pH} 1$ (lactic acid) and $\mathrm{pH} 3.8$ (lactate buffer) were analyzed. No lactate was detected in the irradiated samples at $0.001 \mathrm{M}$ concentration however, currently unidentified new products were detected in these samples. In the samples containing an initial concentration of $1 \mathrm{M}$ lactic acid/ lactate buffer similar new products were observed Figure 7. In these experiments we were able to observe a slow decrease in lactate concentration with increasing dose, the rate of which was almost identical for the $\mathrm{pH} 1$ and $\mathrm{pH} 3.8$ samples.

Currently the new peaks are unidentified. Our collaborators at UNLV are using an HPLC mass spectrometry (HPLC-MS) system to identify the degradation products. They are currently running standards of acetic acid and formic acid since they are expected degradation products. .

A similar method development for the identification of DTPA degradation species was initiated, also using the Shimadzu HPLC with photodiode array detector. A reversed-phase C-18 column from Supelco $(25 \mathrm{~cm}, 4.6 \mathrm{~mm}, 5$ $\mu \mathrm{m})$ was used for the separation. Mobile phase was $50 / 50$ methanol / water at a flow rate of $0.4 \mathrm{ml} / \mathrm{min}$. and the column temperature was $30^{\circ} \mathrm{C}$. An injection volume of $10 \mu 1$ was used and the chromatograms were obtained at 360 $\mathrm{nm}$. It appeared that the retention time of DTPA in this condition was $\sim 6.5 \mathrm{~min}$. However, it was suspected that 2 species were co-eluting at this time and a methodology to resolve these peaks has not yet been developed.

We will continue to develop these separation methods for both the lactate components and DTPA components separately to identify all of the new species that are generated. It is envisaged that HPLC-MS will make product identification much simpler than using the diode array detection. In addition to this we will undertake an investigation on how the $\mathrm{pH}$ of these samples changes as a function of lactate (or DTPA) concentration and absorbed dose. This is important because $\mathrm{pH}$ is an important parameter government TALSPEAK extraction efficiency. 


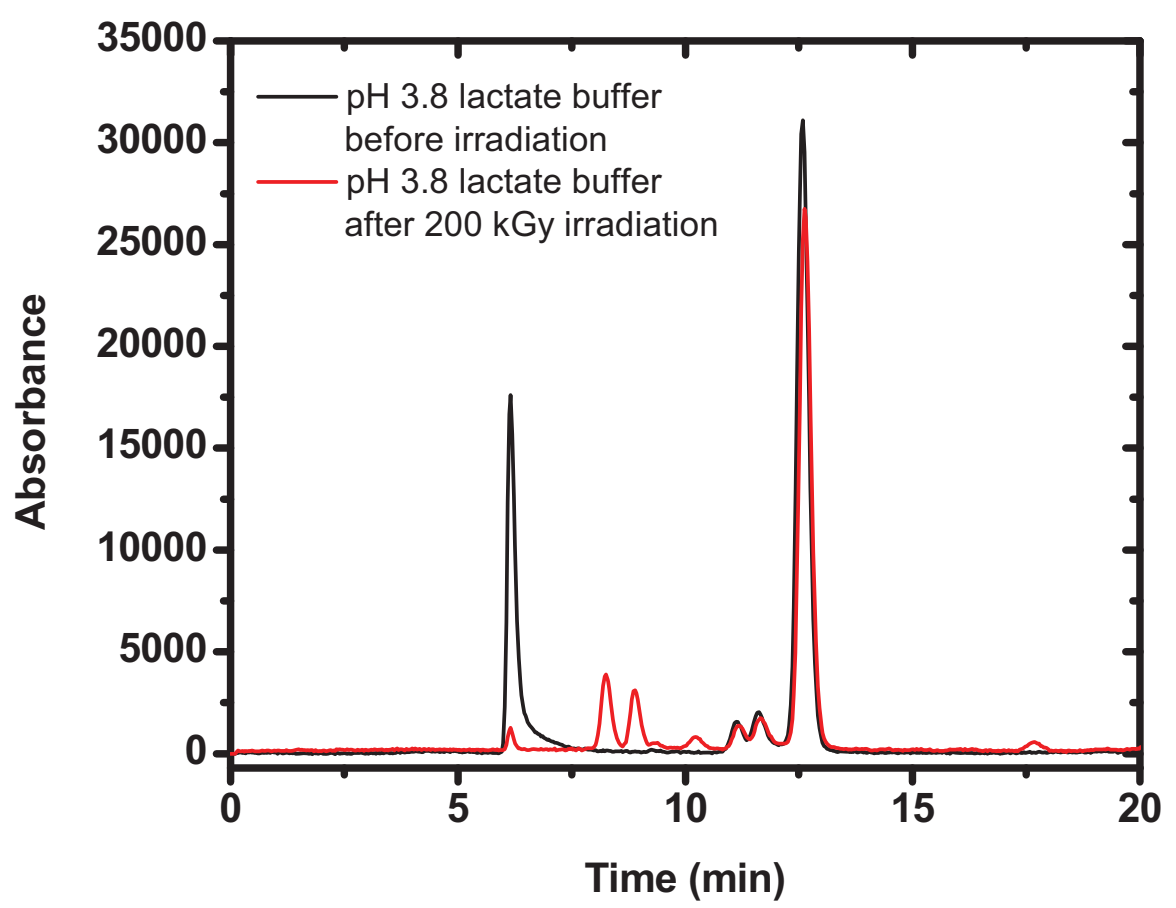

Figure 7HPLC diode array chromatograms for lactate buffer $\mathrm{pH} 3.8,(-)$ with no irradiation and (-) after being irradiated to $200 \mathrm{kGy}$.

\subsubsection{Alpha Radiolysis}

We obtained approvals for using the He ion beam at CNRS in Orleans, France, to perform initial investigations into the role of alpha radiolysis of TALSPEAK components; in particular lactic acid and HEDHP. The following tests were performed and samples shipped back to the INL for analysis.

Solutions of di(ethylhexyl)phosphoric acid (HDEHP) in dodecane with no aqueous phase were irradiated at two different HDEHP concentrations, and two absorbed doses. These were $0.3 \mathrm{M}$ and 1.0 M HDEHP, each irradiated for 80 minutes, and 168 minutes. The purpose of these experiments was to compare the He ion beam results to similar samples at Pacific Northwest National Lab (PNNL) that were irradiated using ${ }^{238} \mathrm{Pu}$. In addition to these experiments solutions of lactic acid in aqueous solution at $\mathrm{pH} 3.7$ with no organic phase were irradiated at two different lactic acid concentrations, each to four absorbed doses. The lactate concentrations were $1 \times 10^{-3} \mathrm{M}$ sodium lactate and $1.0 \mathrm{M}$ sodium lactate, each irradiated for 30,60, 90 and 120 minutes. The $\mathrm{pH}$ of the solutions was adjusted to 3.6 with $\mathrm{HNO}_{3}$. The irradiations were completed in the last week of July 2009, and we are awaiting the stable product analysis of the lactate solutions. Methodologies for identifying HDEHP degradation products are still to be developed. Collaborators at PNNL attempted NMR analysis, unfortunately this was found to be inadequate. ${ }^{11}$

One of the observations that we have been able to make is illustrated in Figure 8. The irradiated aqueous solutions were found to be far more prone to gas generation that the organic solutions of HDEHP. It is not currently understood if this is related to increased solubility of the gas generated in dodecane, or if there was no gas generated in the organic solutions irradiated. It is not currently known if the gas observed in the aqueous solution is $\mathrm{H}_{2}$, which should also be generated in irradiated dodecane, or perhaps a degradation product of the aqueous lactic acid such as $\mathrm{CO}_{2}$. Oxidative radiolysis of organic acids eventually results in the production of $\mathrm{CO}_{2}$. 


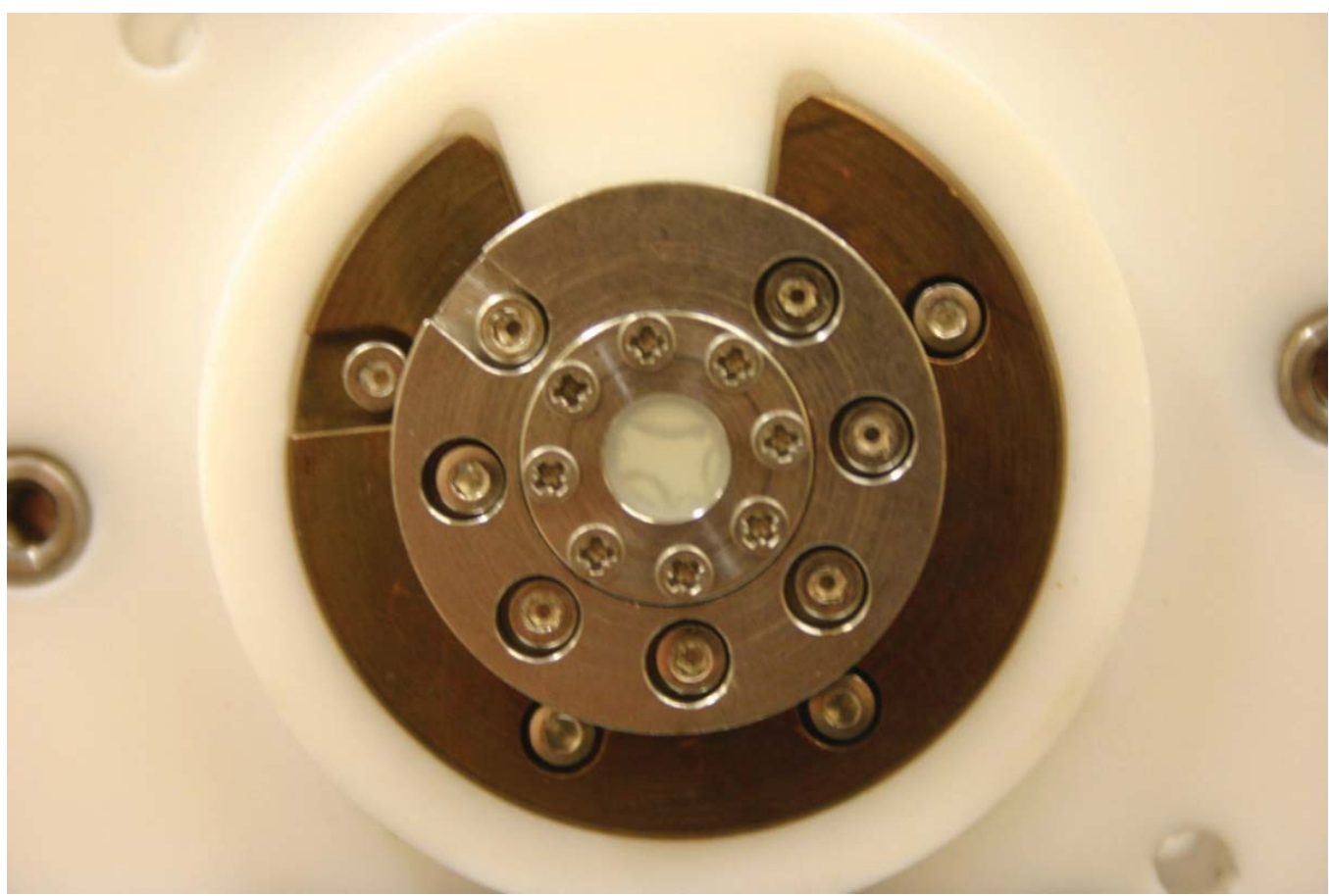

Figure 8. Sample cell after irradiation of $1 \times 10^{-3} \mathrm{M}$ sodium lactate at $\mathrm{pH} 3.7$ for 30 mins. The generated gas is unidentified.

\section{REFERENCES}

1 Weaver, B.; Kappelmann, F. A. TALSPEAK, A new method of separating americium and curium from the lanthanides by extraction from an aqueous solution of an aminopolyacetic acid complex with a monoacidic organophosphate or phosphonate. ORNL-3559, 1964.

2 Nilsson, M.; Nash, K. L. Solv. Extr. Ion Exch. 2007, 25, 665.

3 Tachimori, S and Nakamura, H., Journal of Radioanalytical Chemistry, 1979, 52, 343.

4 Martin, L.R., Mezyk, S.P. and Mincher, B.J. J. Phys. Chem. A, 2009, 113, 141.

5 Mincher, B.J., Martin, L.R. and Mezyk, S.P. J. Phys. Chem. A, 2008, 112, 6275.

6 Buxton, G. V.; Greenstock, C. L.; Helman, W. P.; Ross, A. B. J. Phys. Chem. Ref. Data 1988, 17, 513.

7 Asmus, K. D. Methods Enzymol. 1984, 105, 167.

8 Chin, M.; Wine, P.H. Photochem. Photobiol., A. 1992, 69, 17.

9 Cabelli, D.E.; Rush, J.D.; Thomas, M.J.; Bielski, B.H.J. J. Phys. Chem,. 1989, 93, 3579.

10 Thomas, V.G.; Srivastava, S.B.; Moorthy, P.N. J. Radioanal. Nucl. Chem., 1989, 136, 443.

11 Snow, L.; McNamara, B.; Lumetta, G. and Jones S., NMR Analysis of the TALSPEAK Process Solvent After Alpha-irradiation with Plutonium-238, Poster presented at the Actinides Separation Conference, Reno, NV, 2009. 


\section{INDICATORS OF PROJECT QUALITY AND PRODUCTIVITY}

1. The INL PI (Martin) presented a paper at the $238^{\text {th }}$ National ACS meeting in Washington, DC in August 2009. The paper will be appearing in the resulting symposium book series.

2. The co-PI (Mincher) chaired a session on Radiation Chemistry of Solvent Extraction Ligands at $238^{\text {th }}$ National ACS meeting in Washington, DC August 2009, and was a co-organizer for the symposium Nuclear Energy and the Environment, in which this session took place.

3. A collaboration was initiated with Catherine Corbel of CNRS France, for He ion Beam radiolysis, indicating the international interest in this research.

\section{COLLABORATORS AND PARTICIPANTS}

1. Leigh R. Martin, Idaho National Laboratory, PI.

2. Bruce J. Mincher, co-PI.

3. Richard D. Tillotson, Idaho National Laboratory, performs solvent extraction experiments.

4. Gracy Elias, Idaho National Laboratory, performs HPLC stable product analysis.

5. Stephen P. Mezyk, Cal State Long Beach, kinetic analysis and modeling.

6. Patricia Paviet-Hartmann, University of Nevada Las Vegas, developed methodologies for stable product analysis.

7. Catherine Corbel, CNRS Orleans, He ion beam radiolysis. 